\title{
A nanoscale genosensor for early detection of COVID-19 by voltammetric determination of RNA-dependent RNA polymerase (RdRP) sequence of SARS-CoV-2 virus
}

\author{
Leila Farzin $^{1} \cdot$ Sodeh Sadjadi $^{1} \cdot$ Azarmidokht Sheini $^{2} \cdot$ Elham Mohagheghpour $^{1}$ \\ Received: 26 December 2020 / Accepted: 22 February 2021 / Published online: 10 March 2021 \\ (C) The Author(s), under exclusive licence to Springer-Verlag GmbH, AT part of Springer Nature 2021
}

\begin{abstract}
A voltammetric genosensor has been developed for the early diagnosis of COVID-19 by determination of RNA-dependent RNA polymerase (RdRP) sequence as a specific target of novel coronavirus. The severe acute respiratory syndrome coronavirus-2 (SARS-CoV-2) uses an RdRP for the replication of its genome and the transcription of its genes. Here, the silver ions $\left(\mathrm{Ag}^{+}\right)$in the hexathia-18-crown-6 (HT18C6) were used for the first time as a redox probe. Then, the HT18C6(Ag) incorporated carbon paste electrode (CPE) was further modified with chitosan and PAMAM dendrimer-coated silicon quantum dots (SiQDs@PAMAM) for immobilization of probe sequences (aminated oligonucleotides). The current intensity of differential pulse voltammetry using the redox probe was found to decrease with increasing the concentration of target sequence. Based on such signal-off trend, the proposed genosensor exhibited a good linear response to SARS-CoV-2 RdRP in the concentration range $1.0 \mathrm{pM}-8.0 \mathrm{nM}$ with a regression equation $\mathrm{I}(\mu \mathrm{A})=-6.555 \log [\mathrm{RdRP}$ sequence $](\mathrm{pM})+32.676\left(R^{2}=0.995\right)$ and a limit of detection $(\mathrm{LOD})$ of $0.3 \mathrm{pM}$. The standard addition method with different spike concentrations of RdRP sequence in human sputum samples showed a good recovery for real sample analysis (>95\%). Therefore, the developed voltammetric genosensor can be used to determine SARSCoV-2 RdRP sequence in sputum samples.
\end{abstract}

Keywords Genosensor·SARS-CoV-2 RdRP sequence ·PAMAM@SiQDs

\section{Introduction}

The world is currently witnessing the third large-scale pandemic in the last two decades after the severe acute respiratory syndrome (SARS) in 2003 and the Middle East respiratory syndrome (MERS) in 2012 [1]. The novel pandemic, known as COVID-19, is caused by the very contagious severe acute respiratory syndrome coronavirus-2 (SARS-CoV-2) virus. Due to the emergence and rapid global spread of COVID-19, the quick and accurate detection of virus or disease is increasingly vital to control the sources of infection and prevent the extensive spread of the disease. Despite all the efforts made, there is

Leila Farzin

1farzin84@yahoo.com

1 Radiation Application Research School, Nuclear Science and Technology Research Institute, P.O. Box 11365-3486, Tehran, Iran

2 Department of Mechanical Engineering, Shohadaye Hoveizeh University of Technology, P.O. Box 78986, Susangerd, Iran currently a global lack of reliable, fast, and ultrasensitive diagnostic tools for early detection of COVID-19.

Currently, the diagnostic tests for COVID-19 can be classified into two broad categories, namely molecular diagnostic tests and antibody or serology tests [2,3]. The molecular diagnostic tests include nucleic acid amplification strategies, such as reverse transcription polymerase chain reaction (RTPCR), reverse transcription loop-mediated amplification (RTLAMP), and reverse transcription recombinase polymerase amplification (RT-RPA) [3, 4]. Although RT-PCR is considered the gold standard for COVID-19 diagnosis, it suffers from several limitations. An important issue with the RTPCR test is the risk of eliciting false-negative and falsepositive results [5]. The insufficient viral RNA at the point of detection may lead to false-negative results. Compared to RT-PCR, RT-LAMP is faster and does not require prior RNA isolation from the samples [6]. In addition, this isothermal amplification method requires six independent primers to recognize the target sequence and thus enables better specificity in the amplification [7]. Despite high specificity, RT-LAMP has some problems similar to other molecular detection 
techniques, such as unexpected cross-contamination due to the high amplification efficiency. This contamination may cause to false-positive results [8]. The amplification of nucleic acids using RT-RPA is faster than RT-LAMP, at $37^{\circ} \mathrm{C}$ or less. Although this isothermal amplification method has been reported for the rapidly detection of SARS-CoV-2, there are still some deficiencies in the cost and operability. For example, the RT-RPA requires the participation of three enzymes [9].

The nucleic acid-based biosensors (genosensors) are being developed to improve the limitations of the conventional methods. Along with the advancement in medical diagnosis, the genosensors have become a sensitive and reliable technology for viral detection [10-12]. Among genosensors, electrochemical genosensors have attracted a lot of attention due to their fast response, sensitivity, economical properties, and compatibility with microfabrication technology and simple operation mode which makes them compatible with pointof-care (POC) testing.

The gene-based strategies for SARS-CoV-2 detection have some challenges, especially in terms of primer design [13]. Besides, there is a lack of information about the variety of genetic SARS-CoV-2 in humans and animals. Recently, the WHO recommended RNA-dependent RNA polymerase (RdRP) (oligonucleotide ID: RdRP_SARSr-P2 and RdRP_SARSr-P1), envelope (E), and nucleocapsid (N) genes for the detection of the novel coronavirus [14, 15]. Although these genes were reported as potential targets for the detection of coronavirus, Kakhki and co-workers [16] found out that only one of them (RdRP_SARSr-P2: 5'-CAGG TGGAACCTCATCAGGAGATGC-3') was almost specific for the new coronavirus, and the other introduced probes would detect the other types of coronaviruses. Currently, the gene targets selected by different countries for RT-PCR molecular assays are genetically similar, including RdRP sequence and the open reading frame $1 \mathrm{ab}$ (ORF1ab) sequence $[13,16]$. RdRP, a viral enzyme for viral RNA replication in host cells, plays an essential role in the RNA virus life cycle and has no host cell homolog. The SARS-CoV-2 RdRP sequence recommended by the WHO for detection of COVID19 is very closely related to that of SARS. Specifically, in the selected gene sequences, only three fixed nucleotide bases were different between SARS-CoV-2 RdRP and SARS RdRP [17].

In this article, we focus on the label-free and nucleic acid amplification-free determination of SARS-CoV-2 RdRP sequence (RdRP_SARSr-P2) as a promising target for detection of COVID-19 using the silver ion-hexathia-18-crown-6 complex modified carbon paste electrode (CPE-HT18C6(Ag)). The chemically modified CPEs have received much attention in the electrochemical field [18]. Here, the silver ions in the HT18C6 were used for the first time as an electrochemical probe. HT18C6 is a macrocyclic thio crown ligand that is insoluble in water and can form a fairly stable complex with
$\mathrm{Ag}^{+}$ion in nonaqueous media [19]. It has been used as an excellent neutral carrier for the preparation of Ag-selective electrodes [20] and also for the selective extraction of silver ions into 1,2-dichloroethane phase [21]. As it was clearly pointed out in our previous study [20], this is likely due to the high selectivity of the ionophore for silver ion over other metal ions as well as the rapid exchange kinetics of the resulting complex. Chelating agents with soft coordination sites like sulfur seem to generate great affinity toward transition metal ions, such as $\mathrm{Ag}^{+}$. For the immobilization of probe sequences, CPE-HT18C6(Ag) was further modified with the chitosan and PAMAM dendrimer-coated silicon quantum dots (SiQDs@PAMAM). The SiQDs@PAMAM nanocomposite provides a large surface area and abundant amino groups for the immobilization of DNA probes. For the exploration of new sensitive platforms, a strong tendency toward biomolecules, high surface area, and good electrical conductivity is essential [22]. Generally, the signal amplification in the electrochemical biosensors can be achieved by using nanomaterials, such as quantum dots [23, 24], metal nanoparticles [2, 23], and graphene [2]. The conjugation of PAMAM functionalized nanomaterials with the DNA strands provides efficient nanocomposites for sensing purposes $[25,26]$. Dendrimers are widely used as versatile platforms owing to their excellent properties, such as biocompatibility, uniformity, high-branched structure and large numbers of functional ending groups [27].

\section{Experimental}

\section{Reagents and chemicals}

Bovine serum albumin (BSA, $98 \%$ ), (3aminopropyl)trimethoxysilane (APTMS, 97\%), potassium hexacyanoferrate(III) $\left(\mathrm{K}_{3} \mathrm{Fe}(\mathrm{CN})_{6}, \geq 99.0 \%\right)$, potassium hexacyanoferrate(II) trihydrate $\left(\mathrm{K}_{4} \mathrm{Fe}(\mathrm{CN})_{6} \cdot 3 \mathrm{H}_{2} \mathrm{O}, \geq 99.95 \%\right)$, disodium hydrogen phosphate $(99.95 \%)$, sodium dihydrogen phosphate (99\%), potassium chloride ( $\geq 99 \%$ ), sodium chloride ( $\geq 99 \%$ ), methylacrylate (99\%), ethylenediamine $(\geq 99.5 \%)$, dimethyl sulfoxide (DMSO), maleic anhydride (99\%), chitosan, acetonitrile, diethylether, glutaraldehyde solution (GA, 25\% in water), $N$-hydroxysuccinimide (NHS), 1-ethyl-3-(3dimethylaminopropyl) carbodiimide hydrochloride (EDC), sodium hydroxide, and hexathia-18-crown-6 (HT18C6) were purchased from Sigma-Aldrich (Steinheim, Germany, https:// www.sigmaaldrich.com). The extra pure graphite powder, paraffin oil, silver nitrate, methanol, hydrochloric acid, and 4morpholineethane sulphonic acid sodium salt buffer (MES) were prepared from Merck (Darmstadt, Germany, https://www. merckmillipore.com). Phosphate buffer solution (PBS) was prepared using $0.1 \mathrm{M} \mathrm{Na}_{2} \mathrm{HPO}_{4}$ and $0.1 \mathrm{M} \mathrm{NaH}_{2} \mathrm{PO}_{4}(\mathrm{pH} 7.2)$. 
All selected oligonucleotides, including the SARS-CoV-2 RdRP, SARS RdRP, and E (envelope protein gene) sequences were provided by Faza Biotech Company (Iran) according to the previous reports $[1,16,28]$ as follows:

SARS-CoV-2 RdRP (oligonucleotide ID: RdRP_SARSr-P2): 5'-CAGGT GGAAC CTCAT CAGGA GATGC-3'.

SARS RdRP: 5'-C CAGGT GGAAC ATCAT CCGGT GATGC-3'.

\section{E: 5'-ACAC TAGCC ATCCT TACTG CGCTT CG-3'.}

The probe sequence or receptor of SARS-CoV-2 RdRP was modified at the 5'-terminus with an $\mathrm{NH}_{2}$ group.

\section{Instrumentation}

Cyclic voltammetry (CV), differential pulse voltammetry (DPV), and electrochemical impedance spectroscopy (EIS) were performed using a PGSTAT 302N electrochemical workstation (Autolab, Netherlands, https://www.metrohmautolab.com). The bias potential for EIS was $0.18 \mathrm{~V}$. A conventional three-electrode cell, consisting of a Pt wire as the counter electrode, an $\mathrm{Ag} / \mathrm{AgCl}$ electrode $(\mathrm{KCl}, 3 \mathrm{M})$ as the reference electrode, and a carbon paste electrode (CPE, i. $\mathrm{d}=3.0 \mathrm{~mm}$, Azar Electrode, Urmia, Iran) as the working electrode was utilized. Transmission electron microscopy (TEM) images were taken on an EM10C TEM from Zeiss (Germany, http://www.Zeiss.com) operating at $100 \mathrm{keV}$. Scanning electron microscopy (SEM) studies and energy dispersive Xray spectroscopy (EDX) analysis were performed by a ZEISS EVO 18 analytic microscope (Germany, http://www.Zeiss. com) equipped with an energy dispersive X-ray analyzer. Spectrofluorimetric measurements were performed using an Agilent Cary Eclipse fluorescence spectrometer (Agilent Technologies, USA, https://www.agilent.com). The UV-Vis absorption spectra were recorded on a model Shimadzu UV 2550 (Shimadzu Corporation, Japan, https://www.shimadzu. com). A $780 \mathrm{pH}$ Meter was used for measuring $\mathrm{pH}$ at $25^{\circ} \mathrm{C}$ (Metrohm, Switzerland, https://www.metrohm.com).

\section{Synthesis and characterization of SiQDs}

Silicon QDs were synthesized through a one-step hydrothermal method which offers high temperature and pressure as the procedure reported by Feng and co-workers [29]. In brief, $0.368 \mathrm{~g}$ trisidium citrate was dissolved in $8 \mathrm{~mL}$ deionized water in a flask with sustainable aeration of nitrogen for $20 \mathrm{~min}$ to remove oxygen. Then, $2 \mathrm{~mL}$ APTMS was added into the above solution and then thoroughly stirred at room temperature and ambient pressure for $20 \mathrm{~min}$. The as-prepared solution was loaded into a teflon-lined stainless steel autoclave, which was incubated at $200{ }^{\circ} \mathrm{C}$ for $2 \mathrm{~h}$ and cooled to room temperature. To remove byproducts, the resulting SiQDs colloidal solution was treated by dialysis tubing bag $(1 \mathrm{kDa})$ against double distilled water for $12 \mathrm{~h}$. After that, the colloidal solution was concentrated through a rotary evaporator followed by lyophilization to get brown-colored SiQD powder.

The synthesized SiQDs were characterized by TEM, fluorescence, and UV-vis absorption spectroscopy. The TEM image (Fig. 1a) confirms the formation of quasi-spherical SiQDs with an average size of $\sim 10 \mathrm{~nm}$. It should be mentioned that low contrast in the TEM image is due to the low atomic weight of silicon and nanoscale dimensions of the silicon dots, resulting in poor visualization [30]. The obtained image is similar to previously reported literature by Fan and coworkers [31].

Elemental analysis of the SiQDs using EDX is shown in Fig. 1b, illustrating the presence of $\mathrm{Si}, \mathrm{N}$, and $\mathrm{O}$. Peaks of C and $\mathrm{Cu}$ seen in this spectrum arose due to their integral presence in carbon-coated copper grids used for viewing the samples [30].

The SiQDs colloidal solution observed under a handheld UV lamp with an excitation wavelength of $360 \mathrm{~nm}$ shows intense blue color (Fig. 1c) suggesting the formation of blueemitting SiQDs. Excitation and emission spectra of the resultant SiQDs indicates that the SiQDs possess good photoluminescence properties with symmetrical excitation and emission peaks. The emission maximum of the assynthesized SiQDs is centered at $455 \mathrm{~nm}$ upon excitation at $360 \mathrm{~nm}$.

\section{Synthesis and characterization of PAMAM functionalized SiQDs}

The preparation of amine-terminated generation 3 (G 3) PAMAM dendrimer by divergent technique starting from ethylenediamine core followed by repeated Michael type addition and amidation reaction was described in the ESM.

The carboxyl-containing SiQDs were functionalized with PAMAM by carbodiimide coupling reaction [32]. Briefly, $0.2 \mathrm{~g}$ of SiQDs was dissolved in $5 \mathrm{ml}$ of $10 \mathrm{mM}$ MES buffer (pH 6.5). One millimole of NHS and an equimolar amount of EDC were added to the SiQDs with constant stirring and continued for $1 \mathrm{~h}$ at room temperature to activate the carboxylic acid group of SiQDs. Then, $2 \mathrm{~g}$ of PAMAM dendrimer dissolved in $2 \mathrm{ml}$ of $10 \mathrm{mM}$ MES buffer solution was added slowly to the activated SiQDs with stirring condition. After $16 \mathrm{~h}$ of reaction at room temperature, the solution was purified using a dialysis tubing bag $(3.5 \mathrm{kDa})$ against double distilled water for 3 days with change of water periodically to get PAMAM functionalized SiQDs (SiQDs@PAMAM).

The TEM image shows the morphology of SiQDs@PAMAM (Fig. 1d). The surface zeta potential of SiQDs and functionalized SiQDs was studied by Zetasizer. 

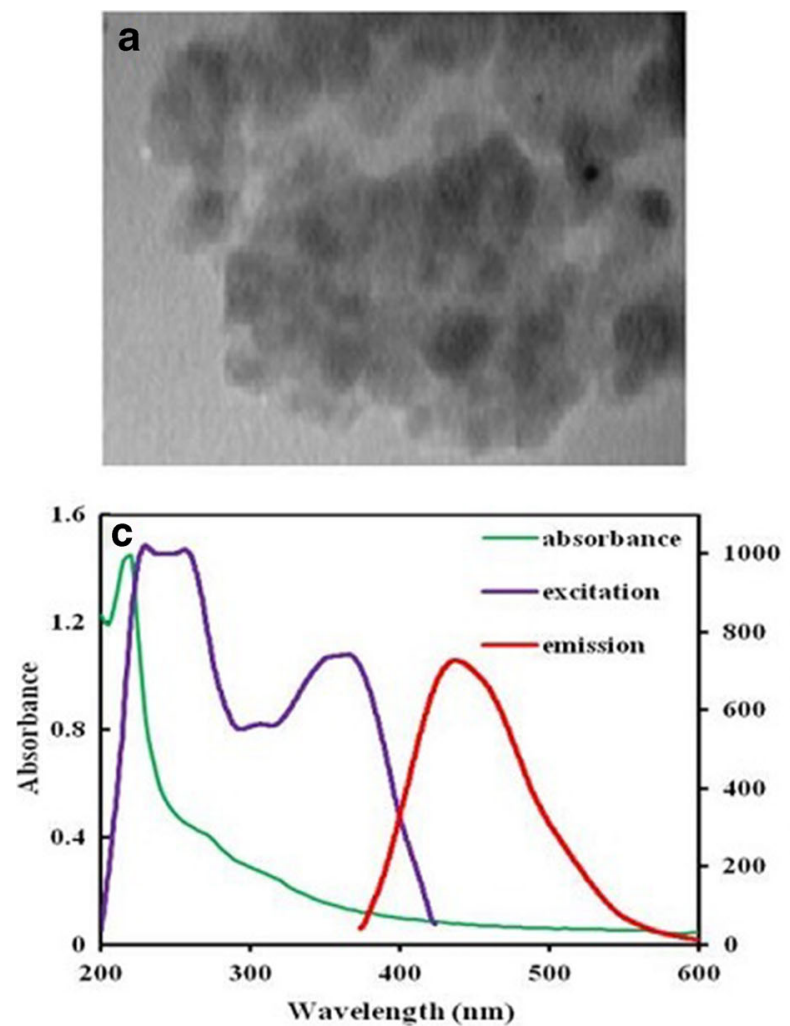
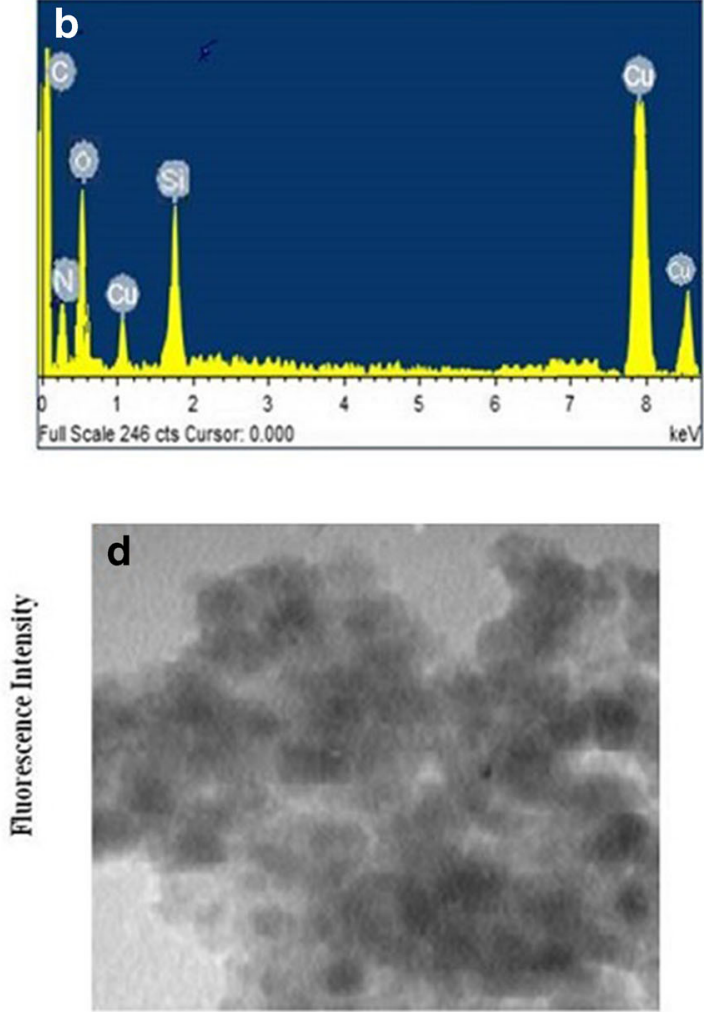

Fig. 1 a TEM image, $\mathbf{b}$ EDX spectrum, $\mathbf{c}$ UV-vis and fluorescence spectra of SiQDs, and d TEM image of SiQDs@PAMAM; the magnification of TEM images was adjusted in $100 \mathrm{KX}$

SiQDs show the negative zeta potential having a value of $2.9 \pm 1.1 \mathrm{mV}$. The negative zeta potential arises due to the presence of carboxylic acid (-COOH) groups on the surface of SiQDs. After conjugation of PAMAM dendrimers, the zeta potential of SiQDs changes to positive $(10.7 \pm 1.0 \mathrm{mV})$ and the magnitude of positive zeta potential gradually increases with an increase in the generation of PAMAM dendrimers.

\section{Development of voltammetric genosensor}

The synthesis of $\left[\operatorname{Ag}\left([18] \operatorname{aneS}_{6}\right)\right]\left([18]\right.$ aneS $_{6}=$ $1,4,7,10,13,16$-hexathiacyclooctadecane) was briefly described in the ESM. In continuation of our research, the CPE-HT18C6(Ag) was constructed under optimum conditions by mixing $55 \% \mathrm{w} / \mathrm{w}$ graphite powder, $15 \% \mathrm{w} / \mathrm{w}$ HT18C6(Ag), and $30 \% \mathrm{w} / \mathrm{w}$ of paraffin oil. For this purpose, $0.055 \mathrm{~g}$ of graphite powder and $0.015 \mathrm{~g}$ of HT18C6(Ag) were mixed in a mortar and homogenized. Then, $30 \mu \mathrm{L}$ of paraffin oil was added and mixed well. The prepared paste was used to fill a Teflon holder. The microstructure of CPE-HT18C6(Ag) was studied by EDX (Fig. 2a). The peaks of S and Ag observed in this spectrum confirm the presence of HT18C6(Ag) in the composition of carbon paste.

The surface of CPE-HT18C6(Ag) was polished on a clean paper until a smooth surface was observed. Then, $0.5 \mathrm{~mL}$ of $0.01 \%$ chitosan in $0.1 \mathrm{M}$ acetic acid was dropcasted on the
CPE-HT18C6(Ag) and dried at room temperature for $1 \mathrm{~h}$. The surface morphology of CPE-HT18C6(Ag) (Fig. 2b) and CPE-HT18C6(Ag)/chitosan (Fig. 2c) were studied by SEM. It can be seen that surface of CPE-HT18C6(Ag) contains a relatively compact carbon paste matrix. After modification, a homogenous layer of chitosan was formed on the electrode surface.

The obtained CPE-HT18C6(Ag)/chitosan was dipped in 5\% GA for $30 \mathrm{~min}$ to activate amine groups for the reaction with primary amines. The activated CPE-HT18C6(Ag)/chitosan was rinsed with distilled water repeatedly in order to remove unreacted GA from the modified electrode surface. For the immobilization of SiQDs@PAMAM on activated chitosan, the activated CPE-HT18C6(Ag)/chitosan was dipped in a $100 \mu \mathrm{L}$ solution of as-synthesized SiQDs@PAMAM for $2 \mathrm{~h}$. Then, the linking of the probe sequence to the amine functionalities of the immobilized dendrimer was performed in a buffered aqueous solution exploiting GA reagent. In the first stage, the CPE-HT18C6(Ag)/chitosan/ SiQDs@PAMAM was dropcasted with $40 \mu \mathrm{L}$ of an aqueous solution of $5 \%$ GA for $30 \mathrm{~min}$. Subsequently, the so treated electrode was washed in MES solution and incubated for $1 \mathrm{~h}$ with $20 \mu \mathrm{L}$ of $10 \mu \mathrm{M}$ solution of the probe oligonucleotide, prepared properly diluting the standard solution in MES buffer. After removal of unreacted sequences by accurate rinsing with MES buffer, a blocking treatment aimed at preventing 
Fig. 2 a EDX spectrum of CPEHT18C6(Ag); SEM images of $\mathbf{b}$ CPE-HT18C6(Ag) and c CPEHT18C6(Ag)/chitosan with magnification of $2.00 \mathrm{KX}$
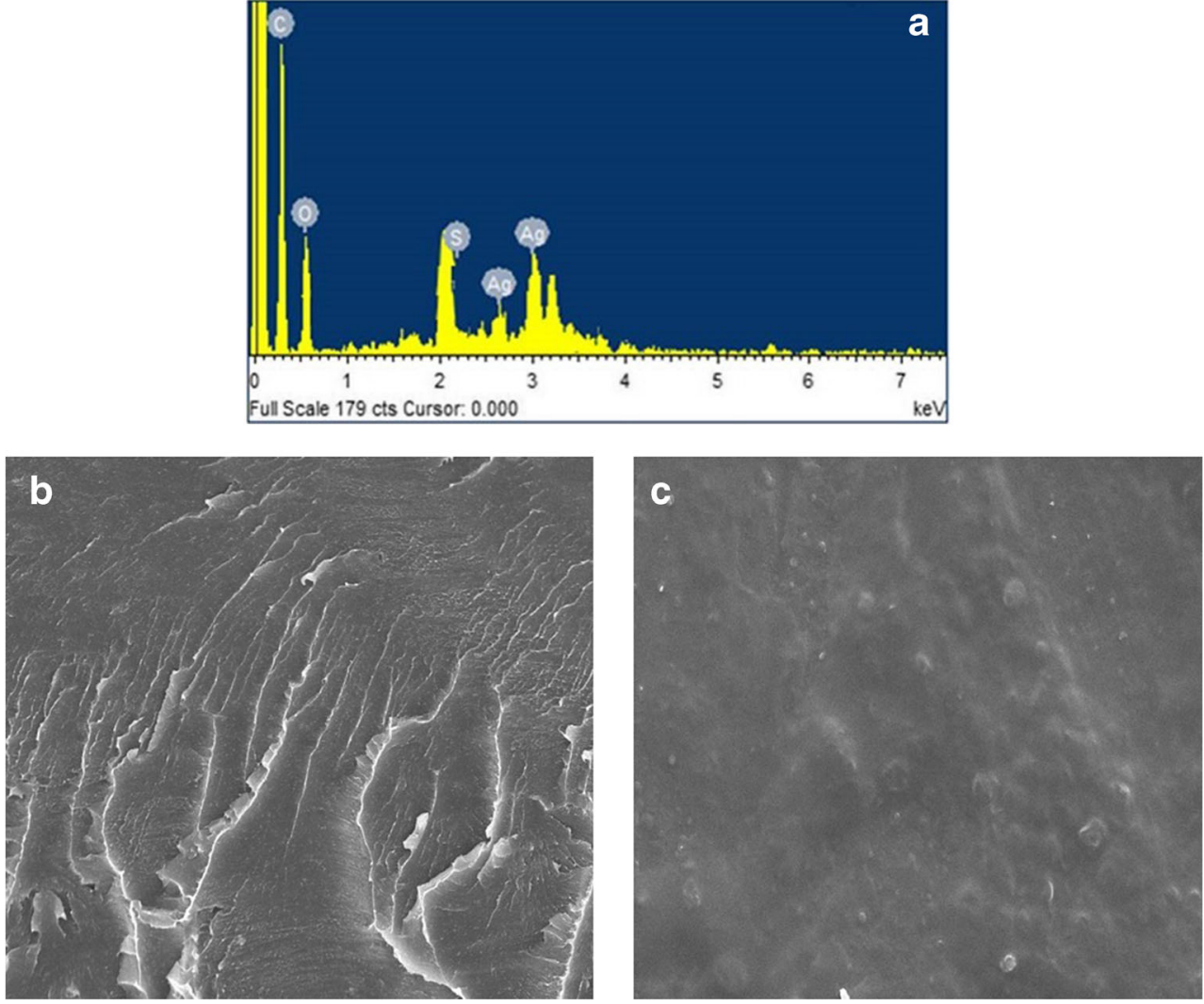

unspecific responses during the sample incubation was carried out by casting $25 \mu \mathrm{L}$ of a $2.5 \mathrm{mg} \mathrm{mL}^{-1}$ solution of BSA dissolved in PBS on the modified electrode for an incubation time of $15 \mathrm{~min}$.

It is important to mention that the stability of immobilized DNA probes on the modified electrode surface is one of the critical factors in the designing of genosensors. We observed the CPE-HT18C6(Ag)/chitosan/SiQDs@PAMAM/ssDNA is more stable than the CPE-HT18C6(Ag)/chitosan/carboxylated SiQDs/ssDNA. The functionalization of SiQDs with PAMAM can lead to more stability of interface compared to unfunctionalized SiQDs. It is because of the entanglement effects of PAMAM on the ssDNA and dsDNA [33]. Nandy and Maiti reported that DNA strands can be entangled on PAMAM dendrimers (G3-G5) [34]. They showed the amine-terminated PAMAM can form a stable complex with ssDNA and dsDNA. It is generally believed that the charge between the positively charged dendrimer and the negatively charged backbones of DNA strands plays a key role in the complex structure [33, 34]. In addition, abundant amino-terminated surface groups of PAMAM dendrimers can be used for the immobilization of aminated DNA probes on the modified electrode through the covalent bonding. Therefore, the electrostatic and covalent interactions of the dendrimer with probe sequences lead to more stability of the designed interface.

After the formation of interface, assays were carried out by incubating CPE-HT18C6(Ag)/chitosan/SiQDs@PAMAM/ probe sequence with $20 \mu \mathrm{L}$ of the target sequence for $25 \mathrm{~min}$ at room temperature. The electrode was then carefully washed to remove unspecifically bound oligonucleotides. All electrochemical measurements were carried out in $\mathrm{pH}$ 7.2 PBS.

Figure 3 presents the schematic illustration of the genosensor fabrication process.

\section{Results and discussion}

\section{Electrochemical characterizaction of step-wise elec- trode surface modification via CV}

Cyclic voltammetry was recorded using the redox signal of silver ion in $0.1 \mathrm{M}$ PBS (Fig. 4A). When the HT18C6(Ag) is mixed with carbon paste, a pair of well-defined redox peaks is observed at 0.42 and $0.26 \mathrm{~V}$ (vs. $\mathrm{Ag} / \mathrm{AgCl}$ ), as a result of oxidation of $\mathrm{Ag}$ to $\mathrm{Ag}+$ (curve a). While the modification of CPE-HT18C6(Ag) with chitosan blocks the electron transfer of silver and decreases redox signals (curve b). As can be seen, a furthermore decrease in the redox peak currents is recorded after modification of electrode surface with SiQDs@PAMAM (curve c). Besides, immobilization of probe sequences on the modified electrode surface leads to a remarkable decline of peak currents in curve d. Finally, as soon as the two sequences are hybridized, the redox signals of silver decrease further due to the thickening of the electron 


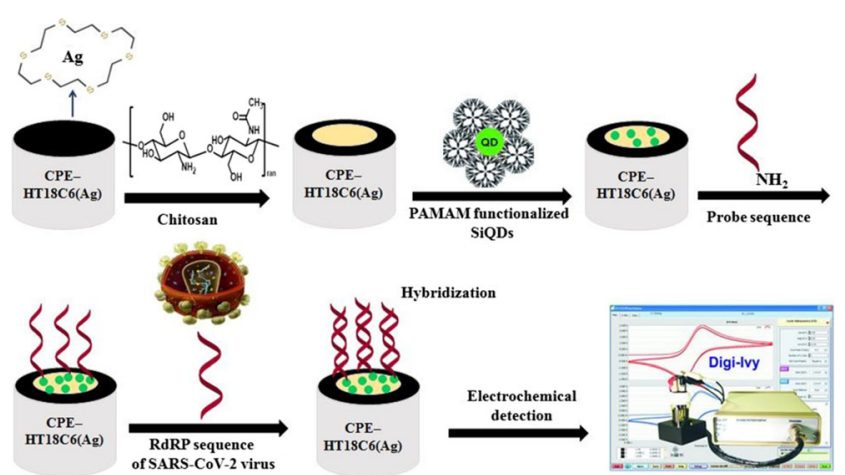

Fig. 3 Schematic illustration of fabrication process of electrochemical genosensor

transfers (curve e). This observation was assigned to the hybridization of probe sequences with SARS-CoV-2 RdRP sequences at the electrode surface which inhibited the redox reaction of silver in CPE-HT18C6(Ag) structure.

The stepwise modification of electrode surface was further studied using the $\mathrm{CV}$ method in the presence of a redox probe $\left(0.1 \mathrm{M} \mathrm{KCl}\right.$ containing $5 \mathrm{mM} \mathrm{Fe}(\mathrm{CN})_{6}^{3-/ 4}$ ) (Fig. 4B). As can be seen, an increase in the redox peak currents is recorded for the electrode after modification with chitosan (curve b) compared to the CPE-HT18C6(Ag) (curva a), due to the positive charge of chitosan. Since the chitosan biopolymers carry the positive charges at the acidic conditions, there is an electrostatic attraction between $\mathrm{Fe}(\mathrm{CN})_{6}^{3-/ 4-}$ and chitosan. Through immobilization of SiQDs@PAMAM on the surface of CPE-HT18C6(Ag)/chitosan, the peak currents are further enhanced due to the electrostatic attraction between positive-charged QDs and $\mathrm{Fe}(\mathrm{CN})_{6}{ }^{3-/ 4-}$ (curve c). After attachment of the probe sequence to the surface of CPE-HT18C6(Ag)/chitosan/SiQDs@PAMAM, the redox peak currents significantly decreased (curve d) related to the presence of phosphate groups in the DNA backbone that repelled the negatively charged probe from the electrode surface [10]. Similarly, the hybridization of SARS-CoV-2 RdRP sequence with the probe sequence led to decreasing the peak currents, owing to the further electrostatic repulsion between redox probe and target sequences (curve e).

EIS method was also carried out to characterize the fabrication process of the genosensor. It is a suitable method for monitoring the changes of the surface features during the fabrication process of electrochemical biosensors [35]. Figure 4 C shows the Nyquist plots of impedance spectra in the process of electrode modification in $0.1 \mathrm{M} \mathrm{KCl}$ containing $5 \mathrm{mM}$ $\mathrm{Fe}(\mathrm{CN})_{6}{ }^{3-/ 4-}$. The impedance of the CPE-HT18C6(Ag) was first obtained (curve a), indicating a very small semicircle at high frequency. When the chitosan solution was dropcasted on the surface of CPE-HT18C6(Ag), the $R_{\mathrm{ct}}$ decreased due to the positive charge of chitosan (curve $b$ ). After the immobilization of SiQDs@PAMAM on the surface of CPEHT18C6(Ag)/chitosan, the electrochemical semicircle became much smaller and close to a straight line (curve c). This is attributed to the electrostatic attraction between $\mathrm{Fe}(\mathrm{CN})_{6}{ }^{3-/ 4-}$ and positive-charged QDs. The assembling of ssDNA probes on the CPE-HT18C6(Ag)/chitosan/ SiQDs@PAMAM led to a significant increase in $R_{\text {ct }}$ (curve d) due to electrostatic repulsion between $\mathrm{Fe}(\mathrm{CN})_{6}^{3-/ 4-}$ anions and the negatively charged probe sequences. Finally, after the incubation of SARS-CoV-2 RdRP sequences with probe sequences, the $R_{\text {ct }}$ showed a further increase (curve e).

\section{Calculation of the electroactive surface area}

In order to increase the active surface area, the number of immobilized probe sequences and finally the sensitivity of the genosensor, the probe sequences were immobilized on the chitosan/SiQDs@PAMAM film. The electroactive surface area for CPE-HT18C6(Ag) and chitosan/SiQDs@PAMAM modified CPE-HT18C6(Ag) was determined from the cyclic voltammograms in solution of $5 \mathrm{mM}$ of $\mathrm{K}_{3}\left[\mathrm{Fe}(\mathrm{CN})_{6}\right]$ containing $0.1 \mathrm{M} \mathrm{KCl}$ at various sweep rates.

According to Eq. (1):

$I_{p}=\left(2.69 \times 10^{5}\right) n^{3 / 2} \mathrm{ACD}^{1 / 2} v^{1 / 2}$

where $i_{\mathfrak{p}}$ is the peak current, $n$ is the number of electrons in the reaction, $A$ is the electrode area, $D$ is the diffusion coefficient of the oxidized form hexacyanoferrate (III), $C$ is the bulk concentration of the oxidized form and $\mathrm{n}$ is the scan rate. From the slope of the $i_{p}$ vs. ${ }^{1 / 2}$ line, the values of $\mathrm{A}$ were determined as $0.099 \mathrm{~cm}^{2}$ and $0.150 \mathrm{~cm}^{2}$ for the CPE-HT18C6(Ag) and chitosan/SiQDs@PAMAM-modified CPEHT18C6(Ag), respectively. Therefore, after the electrode surface was modified with chitosan/SiQDs@PAMAM film, the electroactive surface area greatly increased, indicating that the introduction of interface provides more conduction pathways for the electron transfer of $\left[\mathrm{Fe}(\mathrm{CN})_{6}\right]^{3-/ 4-}$. In addition, chitosan/SiQDs@PAMAM interface offers a large active surface area for immobilization of probe sequences.

\section{Optimization of effective parameters on the genosensor response}

The electrochemical responses of a genosensor can be influenced by several experimental parameters that should be optimized to obtain better performances. One of the effective factors in the electrode response is the amount of HT18C6(Ag) used in the paste composition. By increasing the amount of HT18C6(Ag) in the paste composition, the number of redox probes is increased. However, with a further increased $\mathrm{HT} 18 \mathrm{C} 6(\mathrm{Ag})$ amount, the electrical conductivity of the electrode is decreased [36]. To obtain the optimum value 

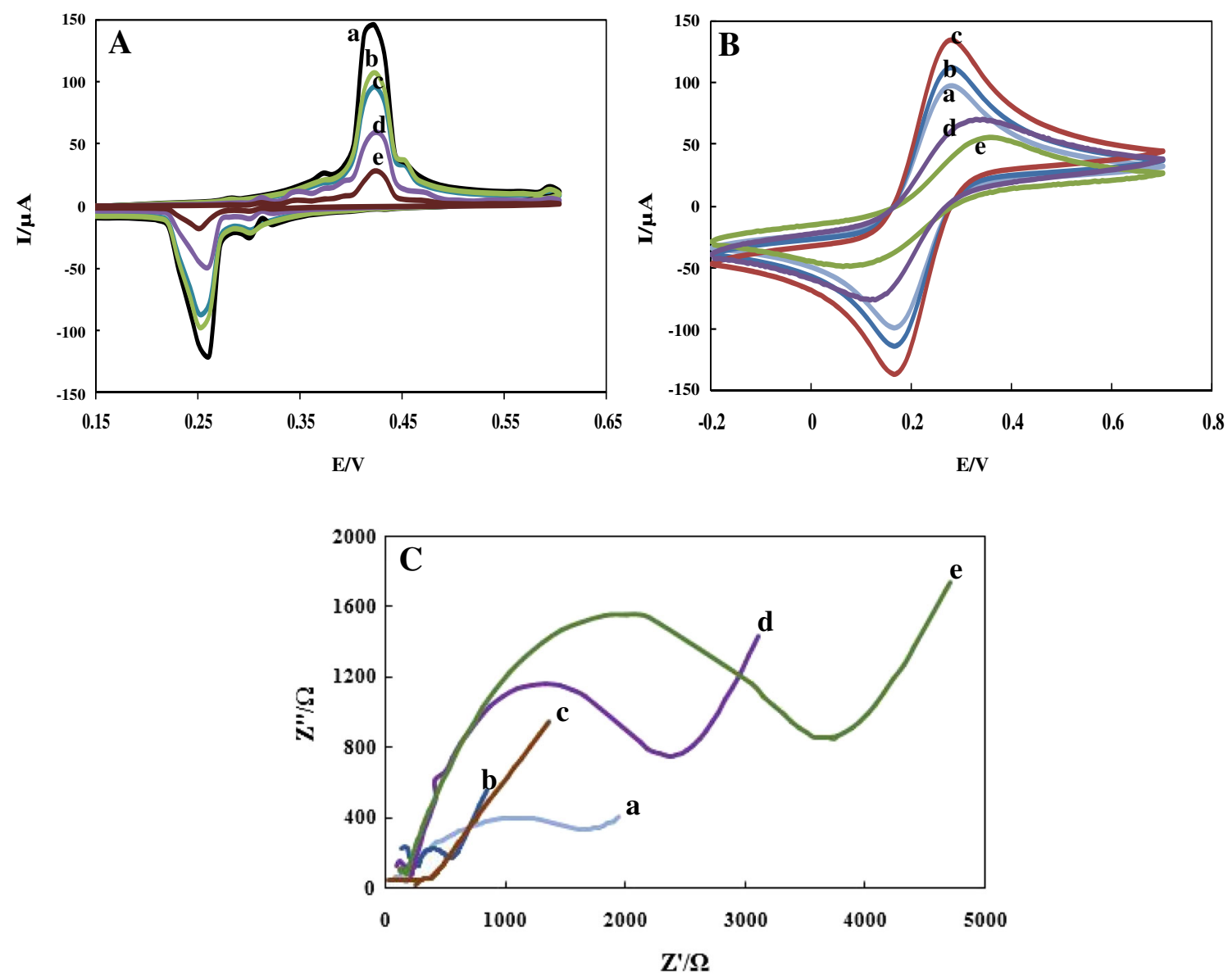

Fig. 4 CVs of CPE-HT18C6(Ag) (a), CPE-HT18C6(Ag)/chitosan (b), CPE-HT18C6(Ag)/chitosan/SiQDs@PAMAM (c), CPE-HT18C6(Ag)/ chitosan/SiQDs@PAMAM/probe sequence (d), CPE-HT18C6(Ag)/ chitosan/SiQDs@PAMAM/probe sequence/SARS-CoV-2 RdRP sequence $(1 \mathrm{pM})(\mathrm{e})$ in A $0.1 \mathrm{M}$ PBS $(\mathrm{pH}=7.2)$ and $\mathbf{B} 0.1 \mathrm{M} \mathrm{KCl}$ containing $5 \mathrm{mM} \mathrm{Fe}(\mathrm{CN})_{6}{ }^{3-/ 4-}$. C Nyquist plots of CPE-HT18C6(Ag)

for HT18C6(Ag), the amount of paraffin oil was kept constant at a $30 \%$ level, while the amounts of HT18C6(Ag) and graphite powder were changed. Results showed the best response was obtained in 55\% graphite powder, $15 \%$ HT18C6(Ag), and $30 \%$ paraffin oil (Fig. 1S).

Among the experimental parameters, the concentration of probe oligonucleotides is an important factor on the response of genosensors. As seen in Fig. 2S, the peak current of redox decreased with increasing concentration of loaded probe oligonucleotides on the surface of modified electrode up to $10 \mu \mathrm{M}$. However, the higher concentrations of probe sequences led to the saturation of electrode surface.

Since the hybridization time is a vital influencing factor on the response of genosensors, the effect of this parameter on the sensor response was also investigated (Fig. 3S). As is obvious, the current response decreased rapidly with increasing hybridization time up to $25 \mathrm{~min}$ and then leveled off at higher periods, suggesting that the formation of hybridized oligonucleotides at the electrode surface has reached a saturation level. (a), CPE-HT18C6(Ag)/chitosan (b), CPE-HT18C6(Ag)/chitosan/ SiQDs@PAMAM (c), CPE-HT18C6(Ag)/chitosan/SiQDs@PAMAM/ probe sequence (d), CPE-HT18C6(Ag)/chitosan/SiQDs@PAMAM/ probe sequence/SARS-CoV-2 RdRP sequence $(1 \mathrm{pM})(\mathrm{e})$ in $0.1 \mathrm{M} \mathrm{KCl}$ containing $5 \mathrm{mM} \mathrm{Fe}(\mathrm{CN})_{6}{ }^{3-/ 4-}$

\section{Analytical performance of signal-off voltammetric genosensor}

The application of CPE-HT18C6(Ag)/chitosan/ SiQDs@PAMAM/probe sequence for the detection of SARS-CoV-2 RdRP sequence was studied based on a signaloff strategy by the DPV technique in the potential range from 0 to $0.8 \mathrm{~V}$ (vs. $\mathrm{Ag} / \mathrm{AgCl}$ ). For this purpose, the prepared nanogenosensor was incubated with different concentrations of the target sequence, and the peak current of silver was measured as a redox probe. Obviously, the addition of target with different concentrations on the modified electrode surface induced different decreases in the peak current of the Ag probe. In fact, the oxidation peak current of silver decreased by adding more increments of SARS-CoV-2 RdRP sequence to the solution (Fig. 5a). Based on such signal-off trend, the genosensor exhibited a good linear response to target in the concentration range of $1.0 \mathrm{pM}-8.0 \mathrm{nM}$ with a regression equation of $\mathrm{I}(\mu \mathrm{A})=$ $-6.555 \log [\mathrm{RdRP}$ sequence $](\mathrm{pM})+32.676\left(R^{2}=0.995\right)(n=$ 
6) (Fig. 5b). The limit of detection (LOD) and limit of quantification (LOQ) evaluated as $3 S_{\mathrm{b}} / \mathrm{m}$ and $10 S_{\mathrm{b}} / \mathrm{m}$ were $0.3 \mathrm{pM}$ and $1.0 \mathrm{pM}$ respectively, where $S_{b}$ is the blank standard deviation and $m$ is the slope of the calibration curve.

The selectivity of the detection largely reflects the performance of the designed genosensor. Thus, the specificity of the CPE-HT18C6(Ag)/chitosan/SiQDs@PAMAM/probe sequence toward different sequences including SARS-CoV-2 RdRP (10 pM), SARS RdRP (50 pM), and E (50 pM) sequences was investigated using DPV technique. The considerable decrease in the peak current of silver was only observed in the presence of the SARS-CoV-2 RdRP sequence, while negligible changes were observed with the other sequences tested (Fig. 6A). In addition, the bar chart of DPV response of CPEHT18C6(Ag)/chitosan/SiQDs@PAMAM/probe sequence in the mixture of target gene $(10 \mathrm{pM})$ and mentioned interferent genes (fivefold) showed a negligible decrease compared with that of modified electrode for target gene (Fig. 6B). Thus, it was concluded that the immobilized DNA probe selectively binds to the target sequence. These outcomes show the selectivity and specificity of the electrochemical genosensor toward the SARS-CoV-2 RdRP sequence.

The relative standard deviations (RSDs) for singleelectrode repeatability and electrode-to-electrode reproducibility were less than $2.7 \%$ and $6.6 \%(n=5)$, respectively. Moreover, the stability was investigated by the electrode immersion in $0.1 \mathrm{M}$ PBS (pH 7.2) for 15 days at the refrigerator $\left(4{ }^{\circ} \mathrm{C}\right)$ and room temperature $\left(25^{\circ} \mathrm{C}\right)$. The constructed DPV aptasensor shows negligible current decay at $4{ }^{\circ} \mathrm{C}$, with RSD of $1.94 \%$; while RSD of $3.78 \%$ was found when the modified electrode was stored at room temperature. So, when not in use, the working modified electrode was stored at $4{ }^{\circ} \mathrm{C}$.

To evaluate the practical application of this sensing strategy, the SARS-COV-2 RdRP gene was determined in the spiked sputum samples. For this purpose, sputum samples prepared from healthy volunteers $(n=3)$ were centrifuged at $5000 \mathrm{rpm}$ (10 $\mathrm{min})$ and then, diluted three times with MES buffer. The standard addition method and DPV technique at positive potential of $\sim 0.42 \mathrm{~V}$ versus $\mathrm{Ag} / \mathrm{AgCl}$ were used to measure target in the sputum samples. The samples were spiked with different concentrations of SARS-CoV-2 RdRP sequence ( $5.0 \mathrm{pM}, 50.0$ $\mathrm{pM}$, and $5.0 \mathrm{nM}$ ). Each sample was analyzed four times, and the corresponding results are shown in Table 1, which discloses a good recovery in real serum samples (> 95\%). Therefore, the voltammetric genosensor can be used to determine SARSCoV-2 RdRP gene in sputum samples.

\section{Comparison with the alternative biosensors for detection of COVID-19}

The LOD of detection tools plays a major role in the early diagnosis of COVID-19. The low LOD of assay methods is desirable to prevent false-negative results. Table $1 \mathrm{~S}$ presents

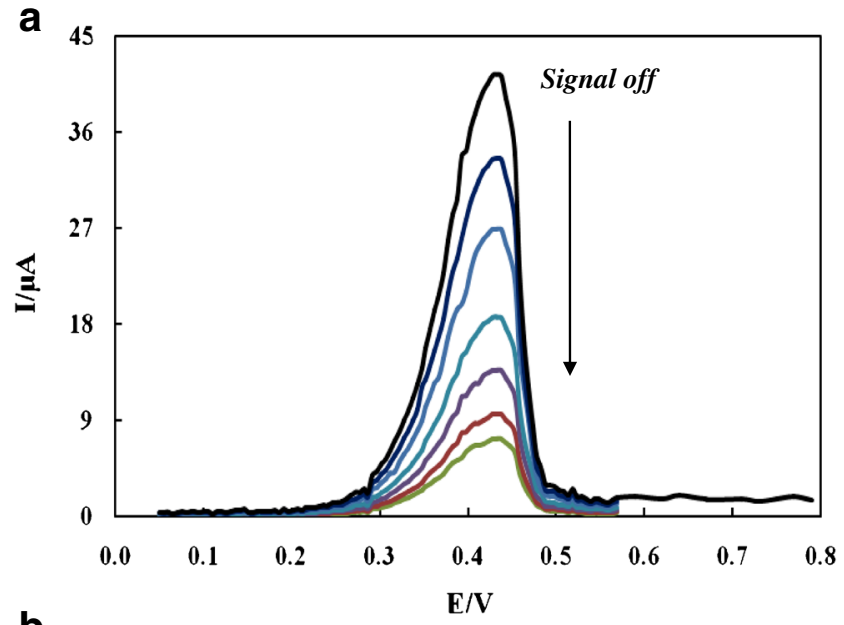

b

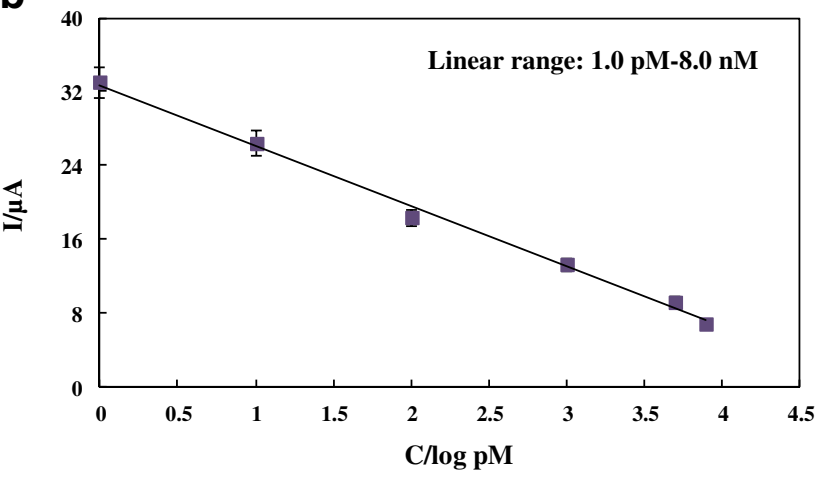

Fig. 5 a DPVs of CPE-HT18C6(Ag)/chitosan/SiQDs@PAMAM/probe sequence in the presence of different concentrations of SARS-CoV-2 RdRP sequence $(0,1 \mathrm{pM}, 10 \mathrm{pM}, 100 \mathrm{pM}, 1000 \mathrm{pM}, 5000 \mathrm{pM}, 8000$ $\mathrm{pM}$ ) in $0.1 \mathrm{M}$ PBS of $\mathrm{pH} 7.2$; $\mathbf{b}$ Calibration curve of $\mathrm{I}_{\mathrm{p}} \mathrm{vs}$. $\log \mathrm{C}_{\mathrm{RdRP}}$

several molecular diagnostic tests based on the nucleic acid amplification for SARS-CoV-2 detection. As mentioned, an important issue with the amplification strategies is the risk of eliciting false-positive results due to the unexpected crosscontamination. In addition to false-negative/positive results, the molecular diagnostics are not intended for POC diagnosis of COVID-19 [8].

Biosensors offer great potential to meet diagnostic requirements and can be used to detect COVID-19-related indicators. The advent of new technologies, such as nanotechnology, smartphones, and microfluidic technology has changed the world of sensors and provided portable biosensing systems for POC diagnostic applications. The biosensors also have the ability to simultaneously identify multiple markers. The simultaneous determination is an important need for the fast detection of multiple analytes present in a sample matrix. For example, areas with local infectious disease epidemics, such as Africa and the malaria epidemic have a high risk of simultaneous outbreaks together with COVID-19. The biosensors can simultaneously identify and screen COVID-19 and other local infectious diseases, serving as an early warning system in resource-poor areas [37]. In addition, these analytical devices can work with low sample volumes. More importantly, 

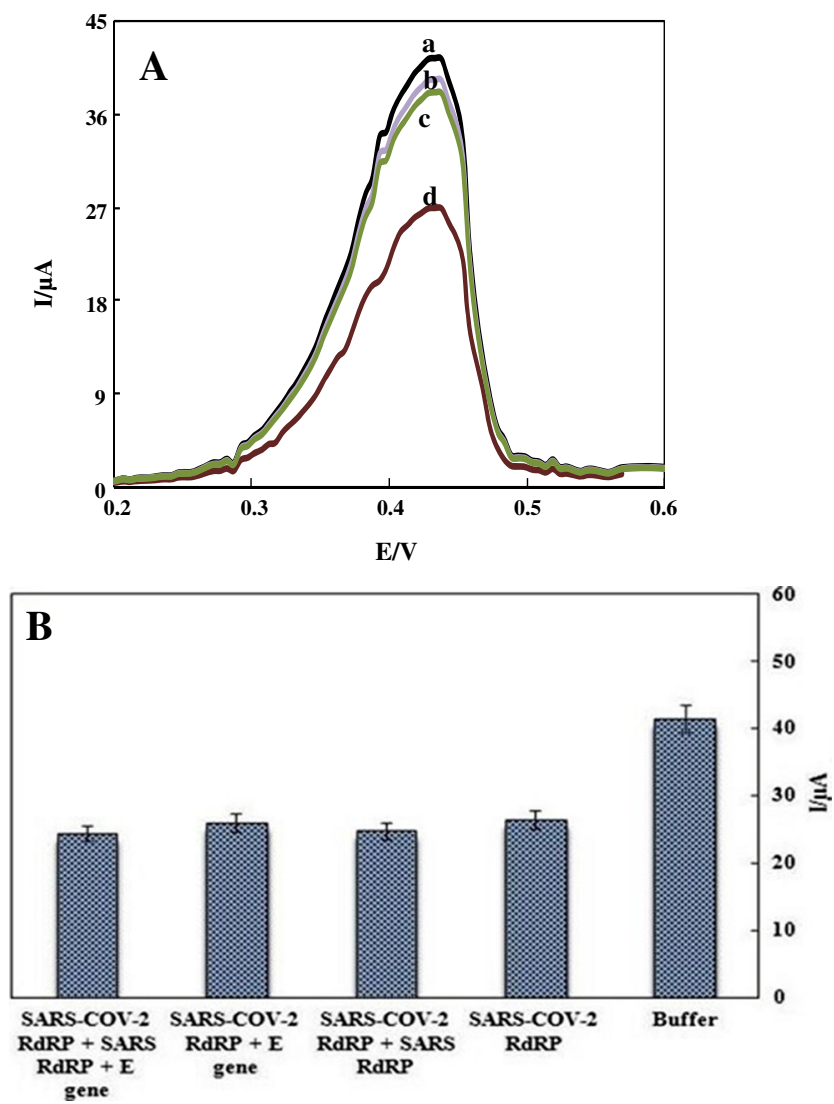

Fig. 6 A DPVs of CPE-HT18C6(Ag)/chitosan/SiQDs@PAMAM/probe sequence in (a) PBS of pH 7.2, (b) E gene (50 pM), (c) SARS RdRP gene $(50 \mathrm{pM})$, and (d) SARS-CoV-2 RdRP gene (10 pM). B Bar charts of DPV responses of CPE-HT18C6(Ag)/chitosan/SiQDs@PAMAM/probe sequence in PBS of $\mathrm{pH}$ 7.2, SARS-CoV-2 RdRP gene (10 pM), and the mixtures of SARS-COV-2 RdRP (10 pM) and other interferent genes (50 $\mathrm{pM})$

biosensors can be used for the label-free and nucleic acid amplification-free detection of DNA/RNA for the sensitive diagnosis of infection [38]. Therefore, ultralow LOD, ability for simultaneous detection, selectivity, and portability of biosensors make them a better choice for COVID-19 diagnosis.

In Table 2, the analytical characteristics of some previously reported biosensors for COVID-19 detection were compared with those of our proposed genosensor [2, 39-45]. The analytical figures of merit of the designed genosensor in this work are acceptable compared to the biosensors reported in the literature for COVID-19 detection. As is obvious, the electrode materials play a critical role in the construction of highperformance electrochemical sensing platforms for detecting ultralow levels of analytes. The PAMAM functionalized SiQDs provide a large surface area and abundant amino groups for the immobilization of DNA probes. In addition, the chitosan/SiQDs@PAMAM nanocomposite, as an efficient bio-interface film, possesses good biocompatibility. To the best of our knowledge, the silver ions in the HT18C6 were used for the first time as an electrochemical probe. The obtained complex was easily mixed with carbon paste. More importantly, no antibody or enzyme was used in our sensing method. Compared with other biosensors that require protein bioreceptors, such as antibodies, the present sensing method demonstrated a simplified need in label-free oligonucleotide probes. The use of nucleic acids can effectively reduce costs and increase the chemical or thermal stability of biosensors. The nucleic acids are in general more stable than antibodies and enzymes and have a longer shelf life.

\section{Conclusions}

The COVID-19 pandemic has created huge damage to society and brought panic around the world. The accurate and reliable diagnosis followed by effective isolation and treatment of patients are pivotal at the early stage of virus breakouts. This is especially true when there is no cure or vaccine available for a transmissible disease, which is the case for the current COVID-19 pandemic. Up to now, more than 100 kits for COVID-19 diagnosis on the market have been proposed, while emerging sensing techniques for SARS-CoV-2 are also investigated. In this article, a signal-off sensing strategy was used for the voltammetric determination of SARS-CoV-2 RdRP sequence (RdRP_SARSr-P2) based on the CPEHT18C6(Ag)/chitosan/SiQDs@PAMAM/probe sequence. For the first time, the silver ions $\left(\mathrm{Ag}^{+}\right)$in the HT18C6 carrier were used as a redox probe. The modification of $\mathrm{CPE}-$ HT18C6(Ag) with nanolayers and then, hybridization of SARS-CoV-2 RdRP sequence with the probe sequence led to decreasing the peak currents of silver. The proposed genosensor exhibits a wide linear range of detection, low LOD, and good selectivity. Despite these advantages, the
Table 1 The obtained results from standard addition method with the spiked different concentrations of SARS-CoV-2 RdRP sequence in sputum samples prepared from healthy volunteers by electrochemical genosensor

\begin{tabular}{lllll}
\hline Sample & Added RdRP sequence & ${ }^{\mathrm{a}}$ Found RdRP sequence & RSD (\%) & Recovery (\%) \\
\hline 1 & $5.0 \mathrm{pM}$ & 4.8 & 1.9 & 96.0 \\
2 & $50.0 \mathrm{pM}$ & 50.4 & 1.0 & 100.8 \\
3 & $5.0 \mathrm{nM}$ & 5.1 & 1.6 & 102.0 \\
\hline
\end{tabular}

${ }^{\text {a }}$ Calculated as a mean of four measurements 


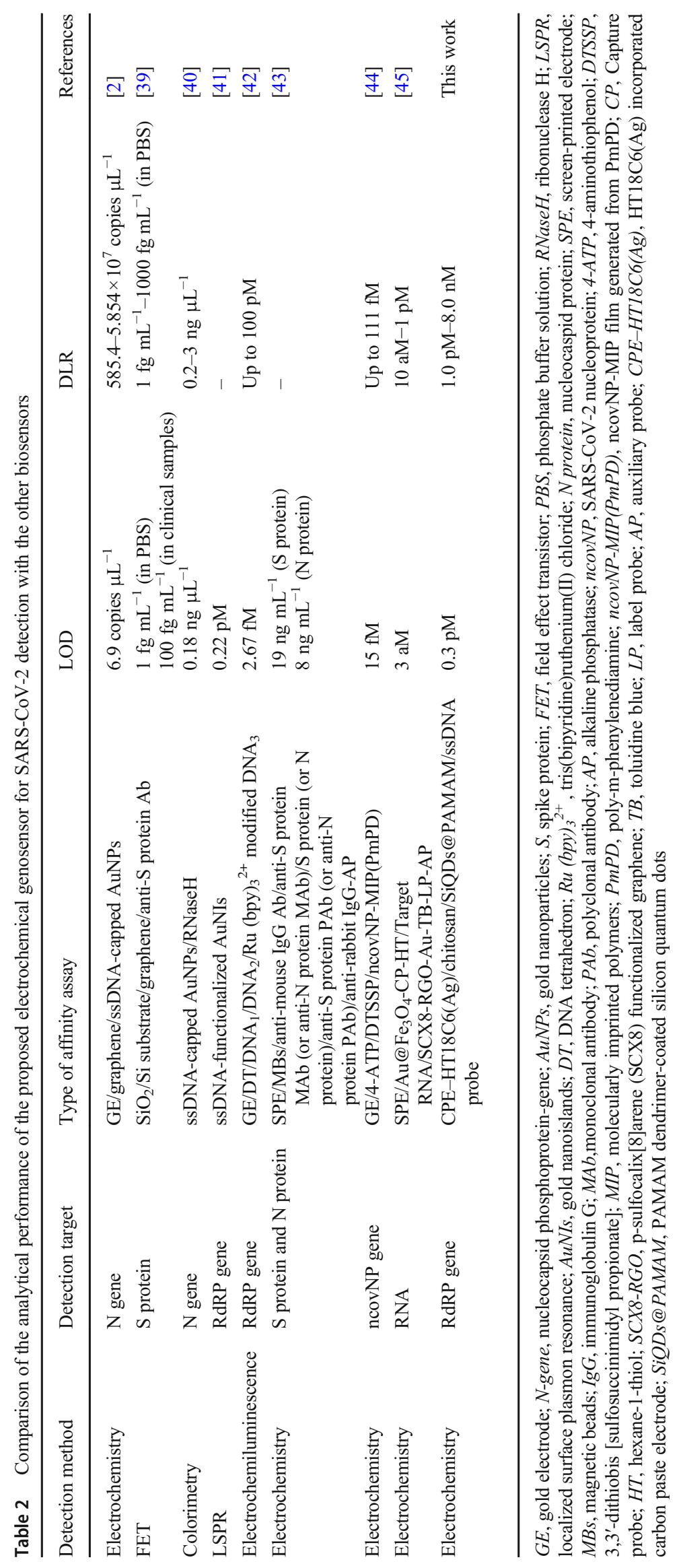


described sensing strategy is time and cost-consuming. In addition, such a design (electrochemical signal-off strategy) has a main limitation of negative signals. To overcome the aforementioned disadvantage, signal-on electrochemical biosensors were duch improved signaling; as the background current observed in the absence of a target is reduced, the gain of such a sensor, at least in theory, increases without limit [46, 47].

Supplementary Information The online version contains supplementary material available at https://doi.org/10.1007/s00604-021-04773-6.

\section{Compliance with ethical standards}

Conflict of interest The authors declare that they have no competing interests.

\section{References}

1. Yang Y, Peng F, Wang R, Guan K, Jiang T, Xu G, Sun J, Chang C (2020) The deadly coronaviruses: the 2003 SARS pandemic and the 2020 novel coronavirus epidemic in China. J Autoimmun 109:102434

2. Alafeef M, Dighe K, Moitra P, Pan D (2020) Rapid, ultrasensitive, and quantitative detection of SARS-CoV-2 using antisense oligonucleotides directed eelectrochemical biosensor chip. ACS Nano 14:17028-17045

3. Chen L, Zhang G, Liu L, Li Z (2021) Emerging biosensing technologies for improved diagnostics of COVID-19 and future pandemics. Talnta 225:121986

4. Lukas H, Xu C, Yu Y, Gao W (2020) Emerging telemedicine tools for remote COVID-19 diagnosis, monitoring, and management. ACS Nano 14:16180-16193

5. Tahamtan A, Ardebili A (2020) Real-time RT-PCR in COVID-19 detection: issues affecting the results. Expert Rev Mol Diagn 2020:1-2

6. Xu L, Li D, Ramadan S, Li Y, Klein N (2020) Facile biosensors for rapid detection of COVID-19. Biosens Bioelectron 170:112673

7. Zhu X, Wang X, Han L, Chen T, Wang L, Li H, Li S, He L, Fu X, Chen S, Xing M, Chen H, Wang Y (2020) Multiplex reverse transcription loop-mediated isothermal amplification combined with nanoparticle-based lateral flow biosensor for the diagnosis of COVID-19. Biosens Bioelectron 166:112437

8. Afzal A (2020) Molecular diagnostic technologies for COVID-19: limitations and challenges. J Adv Res 26:149-159

9. Jiao J, Duan C, Xue L, Liu Y, Sun W, Xiang Y (2020) DNA nanoscaffold-based SARS-CoV-2 detection for COVID-19 diagnosis. Biosens Bioelectron 167:112479

10. Farzin L, Sadjadi S, Shamsipur M, Sheibani S (2020) Electrochemical genosensor based on carbon nanotube/amineionic liquid functionalized reduced graphene oxide nanoplatform for detection of human papillomavirus (HPV16)-related head and neck cancer. J Pharm Biomed Anal 179:112989

11. Farzin L, Shamsipur M, Samandari L, Sheibani S (2020) HIV biosensors for early diagnosis of infection: the intertwine of nanotechnology with sensing strategies. Talanta 206:120201

12. Grabowska I, Malecka K, Stachyra A, Gora-Sochacka A, Sirko A, Zagorski-Ostoja W, Radecka H, Radecki J (2013) Single electrode genosensor for simultaneous determination of sequences encoding hemagglutinin and neuraminidase of avian influenza virus type H5N1. Anal Chem 85:10167-10173
13. Li D, Zhang J, Li J (2020) Primer design for quantitative real-time PCR for the emerging coronavirus SARS-CoV-2. Theranostics 10: 7150-7162

14. Corman VM, Landt O, Kaiser M, Molenkamp R, Meijer A, Chu DKW, Bleicker T, Brünink S, Schneider J, Schmidt ML, Mulders DGJC, Haagmans BL, van der Veer B, van den Brink S, Wijsman L, Goderski G, Romette JL, Ellis J, Zambon M, Peiris M, Goossens H, Reusken C, Koopmans MPG, Drosten C (2020) Detection of 2019 novel coronavirus (2019-nCoV) by real-time RT-PCR. Euro Surveill 25:2000045

15. Moitra P, Alafeef M, Dighe K, Frieman MB, Pan D (2020) Selective naked-eye detection of SARS-CoV-2 mediated by N gene targeted antisense oligonucleotide capped plasmonic nanoparticles. ACS Nano 14:7617-7627

16. Kakhki RK, Kakhki MK, Neshani A (2020) COVID-19 target: a specific target for novel coronavirus detection. Gene Rep 20: 100740

17. Qiu G, Gai Z, Tao Y, Schmitt J, Kullak-Ublick GA, Wang J (2020) Dual-functional plasmonic photothermal biosensors for highly accurate severe acute respiratory syndrome coronavirus 2 detection. ACS Nano 14:5268-5277

18. Roushani M, Valipour A, Saedi Z (2016) Electroanalytical sensing of $\mathrm{Cd}^{2+}$ based on metal-organic frameworkmodified carbon paste electrode. Sens Actuator B-Chem 233:419-425

19. Alberto R, Nef W, Smith A, Kaden TA, Neuburger M, Zehnder M, Frey A, Abram U, Schubiger PA (1996) Silver(I) complexes of the derivatized crown thioether ligands $3,6,9,12,15,18$ hexathianonadecanol and 3,6,9,13,16,19-hexathiaicosanol. Determination of stability constants and the crystal structures of $\left[\mathrm{Ag}(19\right.$-aneS6-OH) $]\left[\mathrm{CF}_{3} \mathrm{SO}_{3}\right]$ and $\left[\mathrm{Ag}(20\right.$-aneS6-OH) $]\left[\mathrm{BF}_{4}\right]$. Inorg Chem 35:3420

20. Mashhadizadeh MH, Shamsipur M (1991) Silver(I)-selective membrane electrode based on hexathia-18-crown-6. Anal Chim Acta 381:111-116

21. Chayama K, Hara K, Tamari Y, Tsuji H, Kusaka Y, Sekido E, Mori Y (1993) In: Logsadil DH, Slater MJ (eds) Solvent extraction in the process industries, vol 1. Elsevier, New York

22. Sarabaegi M, Roushani M, Hosseini H, Hoseini SJ, Bahrami M (2020) Facile synthesis of a covalent organic framework (COF) based on the reaction of melamine and trimesic acid incorporated electrospun nanofiber and its application as an electrochemical tyrosinamide aptasensor. New J Chem 44:14922-14927

23. Valipour A, Roushani M (2017) Using silver nanoparticle and thiol graphene quantum dots nanocomposite as a substratum to load antibody for detection of hepatitis $\mathrm{C}$ virus core antigen: electrochemical oxidation of riboflavin was used as redox probe. Biosens Bioelectron 89:946-951

24. Farzin MA, Abdoos H (2021) A critical review on quantum dots: from synthesis toward applications in electrochemical biosensors for determination of disease-related biomolecules. Talanta 224:121828

25. Zhu N, Gu Y, Chang Z, He P, Fang Y (2006) PAMAM dendrimersbased DNA biosensors for electrochemical detection of DNA hybridization. Electroanalysis 18:2107-2114

26. Niu X, Huang L, Zhao J, Yin M, Luo D, Yang Y (2016) An ultrasensitive aptamer biosensor for the detection of codeine based on a Au nanoparticle/polyamidoamine dendrimer-modified screenprinted carbon electrode. Anal Methods 8:1091-1095

27. Xie J, Wang J, Chen H, Shen W, Sinko PJ, Dong H, Zhao R, Lu Y, Zhu Y, Jia L (2015) Multivalent conjugation of antibody to dendrimers for the enhanced capture and regulation on colon cancer cells. Sci Report 5:9445

28. Yuce M, Filiztekin E, Ozkaya KG (2021) COVID-19 diagnosis - a review of current methods. Biosens Bioelectron 172:112752

29. Feng Y, Liu Y, Su C, Ji X, He Z (2014) New fluorescent $\mathrm{pH}$ sensor based on label-free silicon nanodots. Sens Actuator B-Chem 203:795-801 
30. Wu J, Dai J, Shao Y, Sun Y (2015) One-step synthesis of fluorescent silicon quantum dots (Si-QDs) and their application for cell imaging. RSC Adv 5:83581-83587

31. Fan JW, Vankayala R, Chang CL, Chang CH, Chiang CS, Hwang KC (2015) Preparation, cytotoxicity and in vivo bioimaging of highly luminescent water-soluble silicon quantum dots. Nanotechnology 26:215703

32. Ghosh S, Ghosal K, Mohammad SA, Sarkar K (2019) Dendrimer functionalized carbon quantum dot for selective detection of breast cancer and gene therapy. Chem Eng J 373:468-484

33. Fatemi SM, Fatemi SJ, Abbasi Z (2020) PAMAM dendrimer-based macromolecules and their potential applications: recent advances in theoretical studies. Polym Bull 77:6671-6669

34. Nandy B, Maiti PK (2010) DNA compaction by a dendrimer. J Phys Chem B 115:217-230

35. Valipour A, Roushani M (2017) A glassy carbon immunoelectrode modified with vanadium oxide nanobelts for ultrasensitive voltammetric determination of the core antigen of hepatitis $\mathrm{C}$ virus. Microchim Acta 184:4477-4483

36. Khadem M, Faridbod F, Norouzi P, Foroushani AR, Ganjali MR, Shahtaheri SJ, Yarahmadi R (2017) Modification of carbon paste electrode based on molecularly imprinted polymer for electrochemical determination of diazinon in biological and environmental samples. Electroanalysis 29:708-715

37. Mao K, Zhang H, Yang Z (2020) An integrated biosensor system with mobile health and wastewater-based epidemiology (iBMW) for COVID-19 pandemic. Biosens Bioelectron 169:112617

38. Wu CC, Yen HY, Lai LT, Perng GC, Lee CR, Wu SJ (2020) A label-free impedimetric genosensor for the nucleic acid amplification-free detection of extracted RNA of dengue virus. Sensors 20:3728

39. Seo G, Lee G, Kim MJ, Baek SH, Choi M, Ku KB, Lee CS, Jun S, Park D, Kim HG, Kim SJ, Lee JO, Kim BT, Park EC, Kim SI (2020) Rapid detection of COVID-19 causative virus (SARS$\mathrm{CoV}-2$ ) in human nasopharyngeal swab specimens using fieldeffect transistor-based biosensor. ACS Nano 14:5135-5142

40. Moitra P, Alafeef A, Dighe K, Frieman MB, Pan D (2020) Selective naked-eye detection of SARS-CoV-2 mediated by $\mathrm{N}$ gene targeted antisense oligonucleotide capped plasmonic nanoparticles. ACS Nano 14:7617-7627

41. Qiu G, Gai Z, Tao Y, Schmitt J, Kullak-Ublick GA, Wang J (2020) Dual-functional plasmonic photothermal biosensors for highly accurate severe acute respiratory syndrome coronavirus 2 detection. ACS Nano 14:5268-5277

42. Fan Z, Yao B, Ding Y, Zhao J, Xie M, Zhang K (2021) Entropydriven amplified electrochemiluminescence biosensor for RdRp gene of SARS-CoV-2 detection with self-assembled DNA tetrahedron scaffolds. Biosens Bioelectron 178:113015

43. Fabiani L, Saroglia M, Galatà G, De Santis R, Fillo S, Luca V, Faggioni G, D'Amore N, Regalbuto E, Salvatori P, Terova G, Moscone D, Lista F, Arduini F (2021) Magnetic beads combined with carbon black-based screen-printed electrodes for COVID-19: a reliable and miniaturized electrochemical immunosensor for SARSCoV-2 detection in saliva. Biosens Bioelectron 171:112686

44. Raziq A, Kidakova A, Boroznjak R, Reut J, Opik A, Syritski V (2021) Development of a portable MIP-based electrochemical sensor for detection of SARS-CoV-2 antigen. Biosens Bioelectron 178:113029

45. Zhao H, Liu F, Xie W, Zhou TC, Yang JO, Jin L, Li H, Zhao CY, Zhang L, Wei J, Zhang YP, Li CP (2021) Ultrasensitive supersandwich-type electrochemical sensor for SARS-CoV-2 from the infected COVID-19 patients using a smartphone. Sens Actuator B-Chem 327:128899

46. Wang C, Qian Y, Zhang Y, Meng S, Wang S, Li Y, Gao F (2017) A novel label-free and signal-on electrochemical aptasensor based onthe autonomous assembly of hemin/G-quadruplex and direct electron transfer of hemin. Sens Actuator B-Chem 238:434 440

47. Farzin L, Sadjadi S, Shamsipur M, Sheibani S, Mousazadeh MH (2019) Employing AgNPs doped amidoxime-modified polyacrylonitrile (PANoxime) nanofibers for target induced strand displacement-based electrochemical aptasensing of CA125 in ovarian cancer patients. Mater Sci Eng C 97:679-687

Publisher's note Springer Nature remains neutral with regard to jurisdictional claims in published maps and institutional affiliations. 